\title{
El ocaso de la abundancia: pescadores y pesquerías en Bahía Magdalena en el siglo Xxi
}

\author{
The End of Affluence: Fishers and Fisheries in Bahia Magdalena \\ in the $21^{1 t}$ Century
}

Nemer E. Narchi

EL COLEGIO DE MICHOACÁN, narchi@colmich.edu.mx

Wendi Domínguez

UNIVERSIDAD AUTÓNOMA DE BAJA CALIFORNIA SUR, azulyI@hotmail.com

\section{Demetrio J. Rodríguez Armenta}

SOCIEDAD COOPERATIVA DE PRODUCCIÓN PESQUERA RIBEREÑA Y DE ALTAMAR PESCADORES UNIDOS DE PUERTO SAN CARLOS, S.C.L., demetriorod@hotmail.com

Bahía Magdalena, Baja California Sur, ha sido por mucho tiempo una de las regiones pesqueras más productivas en el país. No obstante, sus productos han ido cambiando a medida que las especies comerciales colapsan. Parte importante de la pesca de escama reportada para Bahía Magdalena es capturada en el Golfo de Ulloa. Lo anterior hace que la situación económica que viven los pescadores se haya agravado con la moratoria pesquera decretada en el segundo semestre de 2016 para inferir la relación entre la pesca y la mortandad de tortuga amarilla (Caretta caretta). En este artículo sostenemos que la pesca en la región surge de manera desorganizada y a partir del colapso del sector agrícola. Mediante el aporte de vińetas que resaltan las historias de vida de los pescadores y el escrutinio de la historia de la pesca en la zona, concluimos que la administración pesquera de la zona obedece a medidas improvisadas y paradigmas obsoletos.

PaLABRAS CLAVE: manejo pesquero, moratoria pesquera, Golfo de Ulloa, agotamiento poblacional, política pesquera.

Magdalena Bay in Baja California Sur has long been one of the most productive fishing areas in Mexico. However, its most important fishery products have undergone marked changes as stocks of commercial species are depleted. A significant proportion of the catch of scaly fish takes place in the Gulf of Ulloa. Recently, the economic situation of local fishers there has worsened due to a moratorium on fishing decreed in the second semester of 2016 to provide an opportunity to study the relation between fishing and the mortality of loggerhead turtles (Caretta caretta). The article argues that the evolution of local fisheries has been chaotic from the outset, when it represented an easy way out from a decayed agricultural industry. Using vignettes that reflect the life histories of fishers, and by carefully scrutinizing the history of fishing in the area, we conclude that fisheries management in the area is based on improvised policies and obsolete paradigms.

KEYwORDs: fisheries management, fishing moratorium, Gulf of Ulloa, stock depletion, fisheries policy.

Fecha de recepción: 1 de diciembre de 2016 / Fecha de aprobación: 14 de julio de 2017 / Fecha de recepción de la versión final: 21 de agosto de 2017 


\section{INTRODUCCIÓN}

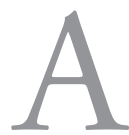

mediados del año 2016, varias poblaciones del Pacífico sudcaliforniano tuvieron que cesar sus operaciones pesqueras en el Golfo de Ulloa con la entrada en vigor del Acuerdo por el que se establece la zona de refugio pesquero y nuevas medidas para reducir la posible interacción de la pesca con tortugas marinas en la costa occidental de Baja California Sur (DOF 2016). La moratoria pesquera que promovía el acuerdo se extendió desde junio hasta septiembre de ese año. Para mitigar los daños al sector pesquero en estos cuatro meses, el gobierno federal puso en marcha un sistema de compensación económica que garantizaba una bolsa de 70 millones de pesos para repartirse entre cooperativistas y permisionarios de escama ubicados en las comunidades de Punta Abreojos, Laguna San Ignacio, El Cardón, El Delgadito, El Dátil, San Juanico, El Chicharrón, Las Barrancas, María Auxiliadora, Ciudad Insurgentes, Santo Domingo, Puerto Adolfo López Mateos y La Poza Grande.

En este artículo, revisamos la (de) evolución de las pesquerías en Bahía Magdalena y el Golfo de Ulloa, desde sus inicios como única opción económica para los jornaleros ante el colapso agrícola de Baja California Sur en la década de los ochenta, hasta el establecimiento del refugio pesquero temporal en junio de 2016. ${ }^{1}$ Para este fin y desde la óptica de la ecología política, ${ }^{2}$ hacemos una reconstrucción histórica de la actividad pesquera en la zona, complementada con datos etnográficos obtenidos a partir de etnografía rápida (Handwerker 2001) ${ }^{3}$ trabajada a lo largo de una semana, cimienta

${ }^{1}$ A pesar de que la gran abundancia en recursos y la diversidad de problemáticas es necesario tratarlas al unísono, pues, las conexiones pesqueras, y por tanto económicas, entre los pescadores de Puerto San Carlos y el Golfo de Ulloa, aunque fuertes, son invisibles a ojos del sistema de registro de capturas pesqueras. Así, los conflictos sociales derivan de la aplicación de medidas de conservación ambiental que se rigen por divisiones políticas y administrativas rígidas y no por dinámicas socioecológicas.

${ }^{2}$ Aunque utilizamos la ecología política como ancla teórica, nos sentimos identificados con las posturas ofertadas por la ecología de eventos (Vayda y Walters 1999). El surgimiento de San Carlos, aunque tocado por la evolución de la política pesquera (véase Alcalá 2003), es producto de una sinergia de factores económicos, tecnológicos y ambientales. Reconocemos en este manuscrito un punto medio entre ambas aproximaciones.

${ }^{3}$ Hay válidos criticismos en contra de la etnografía rápida (e.g., Wolcott 1999), con 
en una relación previa con miembros de la comunidad y que comprende 12 entrevistas semiestructuradas y conversaciones coloquiales sostenidas con pescadores de Puerto San Carlos, Baja California Sur, en mayo de 2016. Las identidades de los pescadores se mantienen en el anonimato por razones de seguridad y acorde con los lineamientos del Código de Ética de la Sociedad Internacional de Etnobiología. Nuestra discusión señala que el tránsito de la pesca, desde un pasado abundante y próspero, hasta la reducción de los stocks pesqueros a puntos casi insostenibles, encuentra su origen en el descuidado manejo gubernamental que ha acompañado a las pesquerías de manera histórica. Hacemos hincapié en la necesidad de mirar a la administración pesquera como un fenómeno que va más allá de lo económico y que se encuentra fuertemente cimentado en lo social. Nuestro fin último es alertar a las autoridades gubernamentales, sector académico y organizaciones no gubernamentales acerca de las posibles consecuencias negativas que el manejo pesquero clásico imprime en la sociedad. Concluimos el manuscrito ofreciendo una reflexión acerca de la imperante necesidad de incluir a las ciencias sociales en los esquemas de manejo pesquero.

\section{Bahía Magdalena-Almejas y Golfo de Ulloa}

Baja California Sur (BCS) es el estado con mayor extensión litoral del país. Sus 2,200 km de costa representan un poco más de $19 \%$ del litoral nacional (INEgi 1995). Como en muchas otras regiones costeras, en BCS el mar no puede entenderse de otro modo que no sea a través de una lente utilitaria que lo vincule con las actividades humanas que ahí ocurren. Estas actividades han dado vida a sistemas de pesquerías asentados en campos y localidades pesqueras distribuidas a lo largo de la costa sudcaliforniana. Las pesquerías, como sistemas productivos, integran variables biológicas, ambientales, tecnológicas, sociales, económicas y políticas, en las que participan

los cuales concordamos. La etnografía nunca debe ser rápida a menos que se tenga una razón válida para ello. En este caso, la expectativa de los pescadores de escama de Puerto San Carlos ante el establecimiento de un súbito acuerdo para establecer una moratoria pesquera que entraría en vigor apenas un mes después de nuestro trabajo de campo. 


\section{Figura I. Ubicación del Golfo de California}
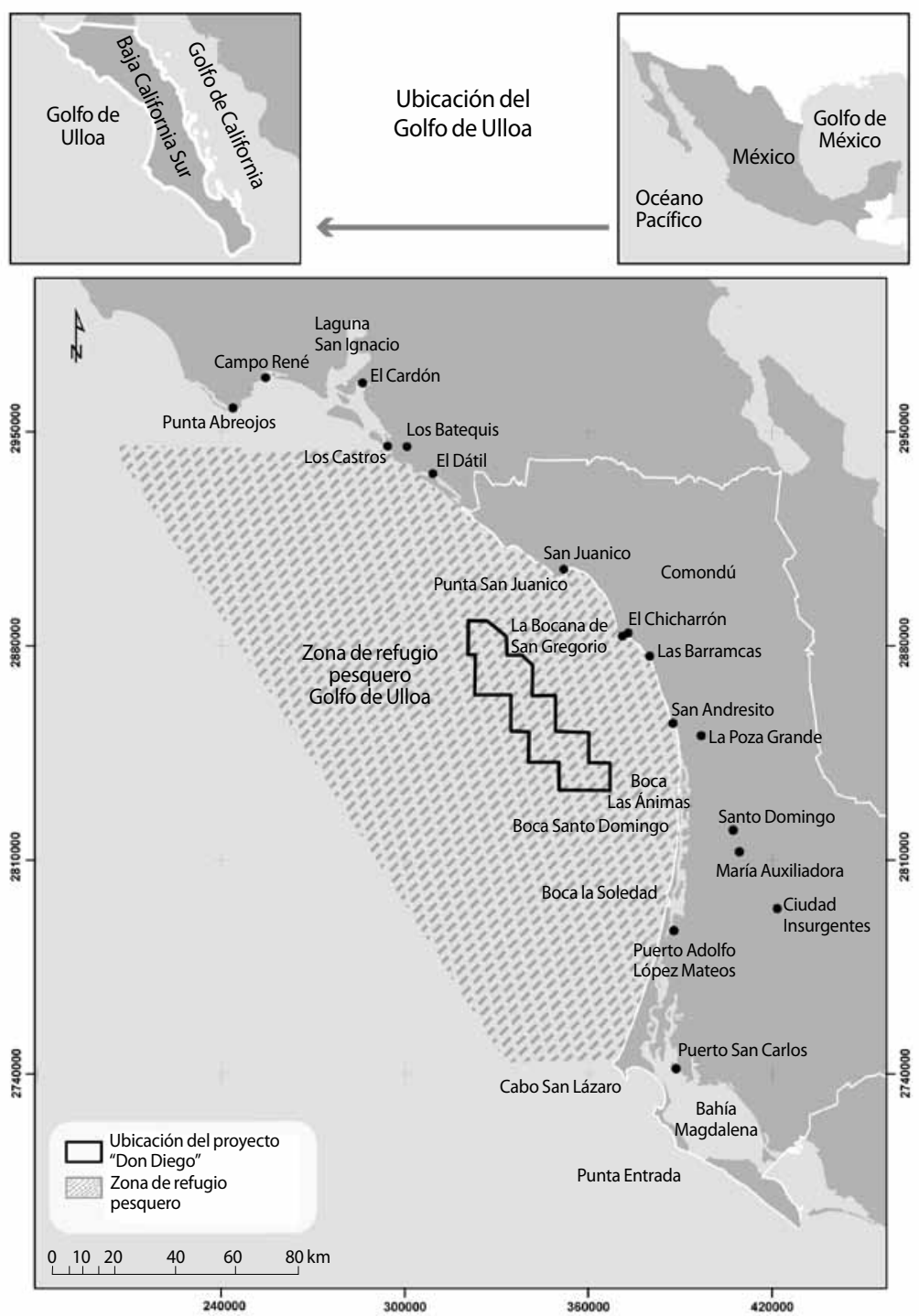

0 Fuente: elaboración propia con base en información vectorial del MGN, 2016. Manifestación de impacto ambiental proyecto "Don Diego". Acuerdo del DOF para la zona de refugio pesquero en el Golfo de Ulloa. Elaboró: geógrafo Jesús Medina R. 
diversos usuarios y actores (Carocci et al., 2009). Las mayores pesquerías en BCS, se desarrollan en las áreas de La Paz y el complejo lagunar de Bahía Magdalena-Almejas (Méndez 2005).

El complejo lagunar Bahía Magdalena-Almejas (BMA) es uno de los más importantes en México y el más extenso de toda la Península de Baja California (véase figura 1). Ubicado en la costa occidental de BCS el complejo lagunar BMA tiene fuertes conexiones ecológicas y oceanográficas con el Golfo de Ulloa (GdU). Este último, localizado al norte del complejo BMA, desde el sur de Punta Abreojos hasta Cabo San Lázaro (sAGARPA 2014), se extiende a lo largo de $348.9 \mathrm{~km}$ (Ramírez et al., 2010).

Las regiones del GdU y BMA son reconocidas por su riqueza en biodiversidad y productividad (Ramírez et al., 2010; SAGARPA, 2014; Bizzarro 2008; Méndez 2005). Los recursos pesqueros de la región contribuyen con más de $30 \%$ de la producción pesquera de México y representan la mayor fuente de empleo para el municipio de Comondú, BCS (SAGARPA 2014). En la región, la pesca ribereña de escama es la más importante seguida de langosta, almeja, raya, abulón, tiburón, cazón, calamar, jaiba, camarón, caracol, pulpo y ostión (Ramírez et al., 2010). La gran biodiversidad de la región es resultado de una dinámica de corrientes que se encuentra bajo la influencia de la corriente de California y la presencia de procesos de surgencia de naturaleza estacional lo que favorece una elevada productividad biológica (SAGARPA 2014; Ramírez et al., 2010).

\section{Descripción económica Bahía Magdalena-Puerto SAN CARLOS}

Dentro del sector pesquero, BCS tiene registrado 8,820 personas ocupadas en esta actividad, de las cuales $42.3 \%$ se ubican en el municipio de Comondú (Ramírez et al., 2010). La zona pesquera de la región comprende de dos grandes comunidades: Puerto San Carlos y Puerto Adolfo López Mateos; así como otros pequeños asentamientos en zonas aledańas. El esquema pesquero predominante corresponde al de pesquerías de pequeña escala (o artesanal) con la interacción entre pescadores agrupados en Sociedades Cooperativas 
de Producción Pesquera (sCPP), permisionarios y pescadores libres, principalmente. Quienes bajo el amparo de permisos otorgados por el gobierno, explotan diversas especies de moluscos: abulón (Haliotidae), almeja chocolata (Megapitaria squalida), almeja catarina ( $A r-$ gopecten irradians), almeja generosa (Panopea generosa y P. globosa), caracol y pulpo (Octopus spp.); crustáceos: langosta, camarón y jaiba; tiburones, rayas y peces (escama) (Ramírez et al., 2010; Funes et al., 2007).

Hablando particularmente de Puerto San Carlos, en 1969 se terminó de construir el muelle con el propósito de distribuir los productos agrícolas que se producían principalmente en el Valle de Santo Domingo (VSD). La delegación municipal de Puerto San Carlos (originalmente conocida como campo pesquero El Sancudal o Moscu), tenía en esa fecha un total de trescientos dos habitantes (Funes et al., 2007). En los ochenta el gobierno suspendió los subsidios al sector agropecuario, decisión que ocasionó la migración de cientos de agricultores (asentados en VSD y originarios del interior del país: Zacatecas, Michoacán y Jalisco, entre otros) hacia sus lugares de origen. Otros más buscaron alternativas económicas que la tierra sudcaliforniana pudiera ofrecer. Una de las principales alternativas fue la pesca, cuyas características en ese entonces pueden describirse como incipientes $y$, hasta cierto punto, rudimentarias. De esta forma, los agricultores se convirtieron en pescadores, asentándose permanentemente en la región.

Se estima que aproximadamente $40 \%$ de la población migró hacia las zonas costeras del estado para dedicarse a la captura de recursos pesqueros (Funes et al., 2007). ${ }^{4} \mathrm{El}$ recorte en los subsidios coincidió con el colapso de la agricultura en el VSD. La agricultura tiene una historia profunda en la región. A partir de 1860 comienza la perforación de pozos, se extiende el área de explotación agrícola, se diversifica la producción y el paisaje se modifica, sobre todo en términos de cambios sensibles en la cobertura vegetal y la saliniza-

${ }^{4}$ Comondú ha fungido como sitio de recepción migrante desde mediados del siglo xx y hasta la década de los ochenta (López 2002). Hoy, cerca de $35 \%$ de los residentes de Puerto San Carlos reportan haber nacido fuera de Baja California Sur (INEgr 2013), pero la zona se ha convertido en un expulsor de población (Gamez, Wilson e Ivanova 2010). 
ción de los acuíferos por efecto de la sobreexplotación de los mismos (Cariño et al., 2011).

El declive del acuífero comienza en los cincuenta y llega a un punto de no retorno en los noventa gracias a la aplicación de políticas federales inadecuadas y a un uso excesivo de las nuevas tecnologías hídricas que, al permitir un ahorro de casi $50 \%$ de agua, se utilizaron para doblar la producción (Cruz Chávez y Ramírez Gómez 2003). Es decir, en lugar de obtener $100 \%$ de la producción algodonera con un volumen cercano a $50 \%$ del agua que se utilizaba antes, los productores de algodón decidieron utilizar $100 \%$ del volumen original.

El colapso agrícola en VSD bien puede considerarse como un fenómeno regional que atañe a todo el Noroeste y que sienta sus bases en el pensamiento de los "agrotitanes", una burguesía agraria de emprendedores que decidió conquistar y modificar el desierto mediante el paquete tecnológico de la revolución verde (Hewitt de Alcántara 1976), que les permitía grandes ganancias con poco riesgo, pero también representaban grandes costos ambientales (véase Narchi et al., 2015). Una suerte de agricultura minera (Chávez-Ortiz 2007) en donde los recursos gestores de la producción agrícola, más que utilizarse, se saqueaban. NAT, un pescador de 71 ańos, recuerda: "Nosotros caímos aquí en el 76. Tenemos 40 años trabajando así. Antes, también fui agricultor y así como nos acabamos la agricultura, también nos estamos acabando la pesca”.

Según INegi (2010) existen 5,538 habitantes en Puerto San Carlos. Y se estimaba que para 2015 la población superó los 6,000 habitantes. No obstante, la población en este puerto varía con respecto a la entrada de ciertas pesquerías debido al alto flujo migratorio, especialmente, durante la temporada de pesca del camarón, la cual se estima que atrae de quinientos hasta dos mil pescadores no residentes de la región, provenientes principalmente de los estados vecinos Sinaloa y Sonora (García-Martínez 2005). Estas variaciones, más el incremento poblacional así como el aumento en la demanda local y extranjera de recursos pesqueros, han provocado un incremento en la presión sobre los recursos naturales de la zona, los cuales se utilizan a una tasa cada vez más alta (Funes et al., 2007). 
Los recursos económicos en Puerto San Carlos son aportados por la industria pesquera $(60 \%)$, la procesadora $(20 \%)$, el comercio $(15 \%)$ y en época de observación de ballenas, el turismo aporta un 5\% (Funes et al., 2007). Respecto al procesamiento pesquero, Puerto San Carlos cuenta con cuatro plantas industriales pesqueras: 1) Conservera San Carlos S.A. de C.V., que utiliza la marca "Calmex" y se dedica al procesamiento de atún en agua (aleta amarilla) y sardina en salsa de tomate. Durante la época de mayor actividad (junio a septiembre), esta empresa emplea entre 500 y 600 personas, de las cuales la mayoría son mujeres; 2) Pesquera México San Carlos S.A. de C.V., planta dedicada a la industrialización y comercialización de sardina (congelado entero); 3) Productos Pesqueros del Golfo de California S.A. de C.V., que procesa calamar seco, deshebrado o congelado; finalmente, 4) SEMAR Internacional S.A. de C.V., que contempla los procesos de fresco y congelado de langosta, escama y calamar, y enlatado de abulón (Méndez 2005). Al ser la segunda fuente de empleo de Puerto San Carlos y al estar ligado directamente a la extracción de recursos marinos como materia prima, estas plantas procesadoras forman parte importante del sustento económico de la región.

\section{SobreEXPlOTACIÓN DE RECURSOS Y PESCA}

INCIDENTAL EN BMA-GU

El régimen de acceso libre (Hardin 1968) que prevaleció en los cincuenta, dio como resultado una explotación sin control de un sinnúmero de recursos naturales, incluidos los pesqueros. Posteriormente, en la década de los sesenta el gobierno empezó a organizar el esfuerzo pesquero al otorgar permisos de pesca comercial, reglamentando embarcaciones y artes de pesca (Funes et al., 2007). Desde entonces, el incremento tanto de permisos como de embarcaciones ha sido constante. Hasta octubre de 2010, en la región del GdU, se tenía el registro de 664 permisos y 1,805 embarcaciones (Ramírez et al., 2010). A estos hay que sumar 325 embarcaciones de pequeña escala con permiso para operar en BMA (Cota-Nieto et al., 2016). Lo anterior, sin contar las embarcaciones o pescadores que realizan su actividad de manera furtiva. 
Las principales pesquerías explotadas son: el camarón, la sardina y la almeja generosa. Con anterioridad la almeja catarina se perfilaba como la pesquería principal. Sin embargo, debido a la sobreexplotación y al consecuente impacto en la especie, los pescadores vieron en la almeja generosa una oportunidad para continuar con sus actividades pesqueras. Además de la almeja catarina, varias poblaciones locales, incluyendo el abulón (Haliotis spp.); la langosta espinosa (Panulirus spp.); y grandes teleósteos demersales (e.g., Serranidae) parecen haber sufrido disminuciones poblacionales, en gran parte, como resultado de la explotación ilegal y no reglamentada (Young 2001). La sobreexplotación de algunos recursos pesqueros y la captura de fauna de acompañamiento en las pesquerías ribereñas en el GdU están amenazando el desarrollo de las comunidades costeras de la región (sAgARPa 2014). Debido a la importancia biológica y socioeconómica y la reciente disminución de varias especies explotadas comercialmente, algunos recomiendan que las pesquerías locales deberían estar mejor controladas y reguladas; fomentar la pesca responsable y así reducir la captura incidental de especies (Bizzarro 2008; SAGARPA 2014). Otra especie que fue sobreexplotada hasta su agotamiento fue el callo de hacha media luna (Pinna rugosa), por parte tanto de los pescadores organizados como de los pescadores libres llamados "aleteros", mediante el buceo a pulmón, en el cual participaban hasta 100 pescadores diariamente por un periodo que duró aproximadamente 10 años, rebasando la capacidad productiva de la población y propiciando su agotamiento.

Dadas las características socioculturales y económicas de la región, los pescadores tienen ingresos moderados, razón por la que cuentan con pocos incentivos para la conservación de los recursos (Ramírez et al., 2010). Es decir, la mayor preocupación de los pescadores es la subsistencia cotidiana, sin importar como incide esta conducta sobre los recursos pesqueros en el largo plazo. Tras el descubrimiento de especies con potencial comercial, se procede a su explotación y consecuentemente a la sobreexplotación, que lleva a la disminución significativa de la especie, la cual se vuelve inaprovechable para las pesquerías y con un gran impacto hacia el ecosistema. 
En la última década, la pesca ribereña ha sido fuertemente relacionada con el varamiento de tortuga caguama o tortuga amarilla (Caretta caretta) en la costa occidental de Baja California Sur (Gardner y Nichols 2001). En esta región existen reportes de avistamiento para cinco de las siete especies de tortugas marinas que viven en México, todas ellas tienen la categoría en peligro de extinción de acuerdo con la NOM-059-SEMARNAT-2001 y otros acuerdos internacionales (Ramírez et al., 2010). No se tiene registro de que la tortuga amarilla anide en el Pacífico mexicano, pero si tiene una zona importante de alimentación en la zona conocida como GdU. Este lugar tiene alta productividad y biodiversidad entre la que se encuentra la langostilla (Pleuroncodes planipes) fuente principal de alimento de la tortuga caguama (Ramírez et al., 2010). De acuerdo con diversos estudios, la tortuga amarilla reportada en la región, proviene de las poblaciones que anidan en el archipiélago japonés (Resendiz et al., 1998; Nichols et al., 2000).

Un buen número de estudios ha reportado una fuerte relación entre la temporada de pesca de escama (mayo-agosto) y un aumento en los varamientos de tortugas muertas (Koch et al., 2006; Peckham et al., 2007, 2008; Gómez Gallardo et al., 2014; Ramírez et al., 2010; Maldonado et al., 2012). No obstante, estudios locales han determinado que la mayoría de las tortugas encontradas en la playas murieron por causas no determinables y sólo 1.8 \% de entre 594 cadáveres encontrados mostró signos claros de heridas por artes de pesca como anzuelos, marcas de red o señales de enredamiento (SAGARPA 2014). Así mismo, en el 2013, se registró el varamiento de 946, de entre las cuales solamente 11 (1.1\%) tenía indicios de interacción con artes de pesca. En 2014 se registraron 174 tortugas muertas, de las cuales $1 \%$ (2) mostró indicios de dańo por actividad humana (SAGARPA 2014). De igual forma, como parte de las actividades programadas por la Procuraduria Federal de Protección al Ambiente (PROFEPA), se ha realizado el monitoreo y registro de varamientos ocurridos en playa San Lázaro. Este esfuerzo registró que menos de $2 \%$ (8/972) de los animales varados presentaban marcas de interacción con pesquerías, al considerar ejemplares en distintos estados de descomposición (Gómez Gallardo et al., 2014). 
A pesar de estos resultados, Organizaciones de la Sociedad Civil (osc) regionales e internacionales señalan fuertemente la actividad pesquera como única responsable de la mortandad de la tortuga. Estas organizaciones afirman que tras algunas indagaciones con pescadores se pudieron identificar algunas posibles causas alternativas: barcos palangreros captura incidental, barcos camaroneros sin redes excluidoras, tiburoneros artesanales, uso de redes de enmalle y volúmenes de captura mucho mayores a los autorizados (Maldonado et al., 2012). Además, estas osc proponen implementar mecanismos de mitigación en la captura incidental en el corto plazo y apelan hacia una presencia más efectiva de las autoridades federales (CONAPESCA, PROFEPA), con el fin de vigilar la captura incidental (Maldonado et al., 2012). Lamentablemente, una visión atomista que vea en el problema una sola causa de mortandad no representa una opción real de solución. Por el contrario, así como atomiza las posibles causas, también altera el potencial de aceptación, adopción e implementación que pueden tener las soluciones paliativas, que trastocan las dinámicas y ciclos sociales y económicos de la región.

Ante tales afirmaciones, otros estudios han abierto el abanico de opciones sobre las posibles causas de la mortandad de tortugas, entre ellas, la investigación presentada por SAGARPA (2014), en la que impera la hipótesis que atribuye la muerte de tortugas amarillas a la presencia de algún tóxico o sustancia radioactiva, por alguna enfermedad (virus, bacteria, etcétera) que pueda afectar a toda la población, por factores ambientales (cambios en las corrientes marinas o presencia de mareas rojas) y, por último, por su interacción con artes de pesca utilizadas por la flota artesanal de la región. Por su parte, Gómez Gallardo y colaboradores (2014) proponen cuatro puntos, de los cuales se rescatan dos, respecto a la causa de la mortalidad de tortugas hay evidencia que le resta peso a la hipótesis de la pesca como principal causa de muerte; $y$, de acuerdo a los experimentos realizados en un animal varado muerto puede provenir de una zona diferente a las utilizadas por la pesquería ribereña de escama. Concluyen así que la interacción de las pesquerías difícilmente puede señalarse como única causa de muerte de la tortuga amarilla. 
Entre los pescadores, incluido nuestro experimentado autor (DRA), se tiene la sensación de que aunque es bien sabido que las tortugas se pueden enmallar en las redes de cazón, corvina y lenguado, entre otros, la mayoría de las tortugas que se enmallan fuera de la bahía son animales que ya se ven enfermos y hasta pareciere que tienen el caparazón blando.

NAT recuerda que:

unos 30 años atrás, la caguama era un negocio. ¿Por qué no se prohibió en ese tiempo? Porque la metieron para el norte. A mí me tocó iba con unos muchachos que pescaban allá agarrábamos 20 caguamas diarias, para matarlas... Pero caguamas de 80 o 90 kilos, había muchas. Aquí la cosa es al revés... toda esa caguama supuestamente que se va, ésa no la matan eso es lo que yo les digo a los biólogos. ¿Por qué quieren engañar a uno? Esa caguama de mayo en adelante comienzan los calores allá que se pone el agua clarita que no puede ni mirar, entonces la caguama hay unas como algas así grandes y la caguama se sube ahí a comer y algo le hace dańo que no madura su buche y no se puede hundir. Por eso viene a dar a esta zona y aquí porque las corrientes la sacan para allá. Entonces la caguama que no pudo sumergirse, esa se murió pero esa no sale para acá, porque no sale porque los vientos jalan para allá. ¿̇A poco no vamos a ver eso, cree que no nos vamos a dar cuenta que las caguamas que varan ahí es por lo mismo que se le seca el buche? Esa ya no se mete para abajo. ¿Y que es lo que pasa? Las corrientes jalan para acá como al 10, pero si las corrientes ahí ... del 38 para afuera agarran a la caguama y ya no se mete para abajo, entonces la corriente las tira a la orilla. Más antes cuando se trabajaba la caguama se agarraba grande la caguama, cuando se trabaja en mayo en adelante, porque de mayo en adelante porque la caguama traía un buche seco y entonces las agarrábamos más fácil. ¿Y cómo los que están investigando no se dan cuenta de eso? ¡Pregúntele a cualquier pescador y les va a decir lo mismo! Si no se necesita ser muy chingón, nomás que uno no sabe las palabras científicas. Si usted me habla con palabras científicas yo no le voy a entender, pero hábleme a como hablamos y vera como si le voy a entender. $j$... La caguama no es porque la están matando! La caguama se vara porque se le seca el buche. Yo me acuerdo que me iba a trabajar la caguama, era de mayo en adelante porque encontrabas 10-15 caguamas todas con el buche seco y se pasaba la 
temporada esa... ¿Y cómo no se van a dar cuenta, mueven intereses y cuando viene el que sea de arriba ya no se la quita uno, así es el problema?

\section{CONSERVACIÓN Y POLÍTICAS PÚBLICAS: LOS ACUERDOS}

A pesar de la falta de mayor número de investigaciones profundas que puedan probar la relación entre la actividad pesquera y la mortandad de tortugas en el GdU, México ha estado en constante amenaza de sufrir un embargo pesquero, es decir, sanciones comerciales por parte de Estados Unidos por no proteger adecuadamente a las tortugas caguamas (Godoy 2015). A través de un informe presentado por el Servicio Nacional de Pesquerías Marinas (NMFs, por sus siglas en inglés), se considera que México no ha adoptado normas mexicanas que sean realmente efectivas para reducir la captura incidental y fomentar la protección de la tortuga amarilla. Según Godoy (2015), la NMFs afirma que en el programa de protección "Acuerdo por el que se establece una Zona de Refugio Pesquero y medidas para reducir la posible interacción de la pesca con tortugas marinas en la Costa Occidental de Baja California Sur" (Dof 2015), el área de restricción tiene alcances geográficos limitados, las restricciones a las redes sólo son aplicables en los meses de mayo a agosto y el tamaño de malla especificado podría reincidir en la captura y mortalidad de las tortugas.

En este primer acuerdo (DOF 2015), la restricción pesquera (únicamente de escama) aplicaba en una superficie de $8,848.2 \mathrm{~km}^{2}$ con temporalidad de 2 años y se establecía como "Área Específica de Restricciones Pesqueras" una superficie de $2,511 \mathrm{~km}^{2}$ donde se podían llevar a cabo actividades pesqueras únicamente con las artes de pesca autorizadas específicamente en los permisos o concesiones de pesca comercial. Al ańo siguiente fue publicado el "Acuerdo por el que establece la zona de refugio pesquero y nuevas medidas para reducir la posible interacción de la pesca con tortugas marinas en la Costa Occidental de Baja California Sur" (Dof 2016), donde no sólo se amplía la Zona de Refugio Pesquero (ZRP), sino que se suspende completamente cualquier actividad de pesca con embarcaciones mayores y menores. La ZRP parcial temporal se extiende a 
una superficie de $19,934 \mathrm{~km}^{2}$ y dentro de la zona de refugio se establece como Área Específica de Restricciones Pesqueras la superficie de $7,244 \mathrm{~km}^{2}$. El periodo de suspensión comenzó en junio de 2016 y se prolongó hasta el 30 de septiembre del mismo año (Dof 2016).

La suspensión pesquera pretende demostrar que el sector pesquero es completamente ajeno a la mortandad de tortugas marinas (SAGARPA 2016b), como declara el gobierno federal mediante un comunicado sobre la veda temporal. El comunicado específica que se resarciría el daño económico a los pescadores afectados, el cual lleva por nombre "Proyecto estratégico para implementar acciones que contribuyan a mitigar el efecto de la restricción normativa en la actividad pesquera en la región del Golfo de Ulloa, BCS”. La compensación total sumó setenta millones de pesos, recurso que se hizo llegar directamente al pescador a través de un padrón de beneficiarios autorizado conjuntamente por el Gobierno del Estado y CONAPESCA (SAGARPa 2016b).

El esquema de indemnización causó gran preocupación entre los pescadores de Puerto San Carlos. A partir de las entrevistas realizadas en mayo de 2016, se mencionó en varias ocasiones que más de la mitad de los pescadores activos de Puerto San Carlos son pescadores libres. Es decir, sin ningún permiso individual o por cooperativa pesquera que los ampare. Además, según los acuerdos publicados en el Dof en el 2015 y 2016, la comunidad de Puerto San Carlos no entra dentro de los parámetros de elegibilidad para acceder a la indemnización correspondiente por no ser considerada dentro de la zona de influencia. Durante las entrevistas, los pescadores expresaron su preocupación ya que para poder ser candidatos al apoyo gubernamental, sus avisos de arribo pesquero tenían que especificar el área de pesca dentro o en las inmediaciones del GdU. Lamentablemente, en los avisos de arribo (instrumento oficial en que el productor reporta a la autoridad los resultados de la producción pesquera) no existe un mecanismo que permita verificar la información que se registra (Ramírez et al., 2010). Nuestros entrevistados mencionaron que por falta de tiempo o capacitación, sus arribos eran elaborados por terceras personas y solamente especificaba BMA como su área de pesca. 
Según Ramírez y colaboradores (2010), CONAPESCA reconoce en la región del GdU 27 lugares de desembarco, desde Punta Abreojos hasta Puerto Adolfo López Mateos y Bahía Magdalena. No se hace mención oficial de Puerto San Carlos, pues, está dentro de BMA. Según SAgarpa (2016a), las comunidades pertenecientes al GdU son: Punta Abreojos, Laguna San Ignacio, El Cardón, El Delgadito, El Dátil, San Juanico, El Chicharrón, Las Barrancas, María Auxiliadora, Ciudad Insurgentes, Santo Domingo, Puerto Adolfo López Mateos y La Poza Grande. Y se mencionan los siguientes requisitos para acceder al proceso de indemnización, a saber (SAGARPA 2016a):

- Ser de las localidades enlistadas.

- Contar con registros de producción correspondientes a las últimas cinco temporadas de pesca.

- Haber participado en las acciones de ordenamiento pesquero en la región del Golfo de Ulloa, BCS.

- Contar con un permiso de pesca comercial vigente para escama o tiburón con zona de pesca en la región del Golfo de Ulloa, BCS.

- Para infraestructura productiva, contar con permiso para Acuacultura de Fomento vigente.

- IFE, CURP, RFC, Comprobante de domicilio, Acta Constitutiva, etcétera.

A la luz de estos requisitos y tomando en cuenta las entrevistas realizadas a pescadores de Puerto San Carlos en mayo del 2016, ninguno de los pescadores de esta comunidad puede acceder a la indemnización. Así, era perceptible un aumento en la suspicacia para con la implementación de la veda pesquera, a la que se atribuye una finalidad que va más allá de la conservación de la tortuga amarilla.

Los tiempos entre el posible embargo pesquero, los acuerdos y el proceso de indemnización coinciden con el interés extranjero de extraer fosfato de manera subacuática mediante la empresa estadounidense "Minera Don Diego". La falta de información y la especulación de los pescadores dio pie a una serie de comentarios sobre la veda pesquera, que una vez implementada, permitiría la exploración y quizá explotación del fondo marino. Este tipo de dragado de mi- 
nerales alberga consecuencias ecológicas potencialmente más catastróficas que la propia mortandad reportada de tortugas marinas.

\section{Pescadores libres 5}

Parte importante del esquema de compensaciones resultante de la moratoria pesquera residía en repartir las compensación entre los socios de las cooperativas y los permisionarios de escama. CONAPESCA (2014) ha calculado que los afectados por la moratoria son unas 950 embarcaciones menores y 1,764 pescadores ribereños. Sin embargo, el mismo documento sobreestima el número de embarcaciones y pescadores en un $10.5 \%$ con la finalidad de capturar en su muestreo a los pescadores tradicionales y libres.

Los pescadores libres son sujetos cuyas relaciones de trabajo gozan de un carácter líquido. Es decir, ofrecen sus servicios a diversos equipos de pesca según les convenga, razón por la cual estos pescadores no están registrados como miembros de ninguna cooperativa pesquera $y$, muchas de las veces, tampoco son permisionarios. Los pescadores libres no tienen prueba de relación laboral en el sector pesquero. Los arreglos que hacen con diversos equipos de pesca son contratos verbales que gozan de liquidez y están gobernados por un carácter efímero. A lo largo de nuestras entrevistas, quienes colaboraron con la investigación opinaron, a pesar de no ponerse de acuerdo en una cantidad exacta, que la fuerza pesquera en San Carlos está integrada, en su mayoría, por pescadores libres.

UJA, un pescador libre, menor de 40 años, dijo estar muy afectado por la moratoria pesquera. Con otros dos pescadores compró un barco que le representaba una oportunidad de mejores ingresos, pero dadas las condiciones impuestas, prácticamente de la noche a

\footnotetext{
${ }^{5}$ Existe una heterogeneidad económica y jurídica que separa a los pescadores en varios grupos: cooperativistas, permisionarios y libres (Gatti 1986). Los primeros, idealmente, constituyen su fuerza de trabajo a partir de miembros de la cooperativa. Los segundos, tienen bajo su mando a pescadores asalariados. Los libres no tienen un contrato fijo y ofrecen su fuerza de trabajo y medios de producción a las cooperativas, los permisionarios o las redes de pesca furtiva. La pesca furtiva no representa cárteles bien establecidos, aunque los hay. Por el contrario, la pesca furtiva tiene cabida en cada uno de los grupos de pescadores aquí representados.
} 
la mañana, por la moratoria pesquera, el barco, más que una herramienta de trabajo, ahora le resultaba una carga que no había imaginado. El relata:

Yo el año pasado hice un inversión de este barquito con ese motor pues siempre me lleve 100 mil pesos y pues hasta ahorita no he sacado más que para comer. Los gastos no los saqué y entonces hoy viene una temporada buena. Haz de cuenta, pues, mayo, junio, julio, agosto se arrima el pescadito... Anduvimos batallando todo estos tiempos y lo que estamos viendo es que verdaderamente nos va [a] afectar muy duro a nosotros por la inversión y aparte por el trabajo pues de que ahí era nuestra área de trabajo. Ahorita, en estos tiempos, [en la bahía] ha estado escaseando un poco la producción y pues [el Golfo de Ulloa] era para donde salíamos nosotros a pescar... Nos va afectar mucho económicamente a nuestras familias y somos tres los que trabajamos en esa embarcación, entonces siempre nos daña somos tres familias que, como se dice, haz de cuenta que le llaman el Golfo de Ulloa lo que es de ahí de San Lázaro para allá pero los de Puerto San Carlos vamos a pescar para allá. Entonces si nos está afectando y no se nos está tomando en cuenta, menos como dice pues como somos pescadores libres que no tenemos algo que diga usted no. Pues como dicen: se le va apoyar a las personas que tengan arribos.

La moratoria pesquera, que sólo reconoce a las cooperativas y permisionarios, deja fuera de toda compensación económica a los pescadores libres. Al no ser sujeto de indemnización y no poder realizar ninguna otra actividad productiva, sea por las condiciones áridas del entorno o porque actividades como el turismo y la pesca deportiva corresponden a temporadas cortas (Young 1999) distintas a las de la veda, los pescadores libres quedan en un estado de indefensión y vulnerabilidad. También es probable que la medida genere cierto encono y animadversión hacia los planes y las especies que se pretende proteger. UJA lo sintetiza muy bien cuando dice: "A mi la caguama nunca me dio de comer".

La presencia de pescadores libres se explica por dos causas principales: 1) Los procesos demográficos de la península, que están conectados al flujo migratorio hacia Estados Unidos y a un flujo 
migratorio interno en donde la gente se mueve en el noroeste según le convenga económicamente; 2) La falta de mano de obra que surge como producto de una necesidad apresurada por conformar las cooperativas. ZA, un pescador explica que la cooperativa necesitaba mantener 5 o más socios “...y ya, metimos a la esposa de cada uno de nosotros, pero ellas no van a marea”.

\section{Aviso de arribo: el otro problema de la compensación}

Parte fundamental de la planeación y administración pesquera se basa en los datos que se obtienen del informe de arribo. Para la mayoría de los pescadores de San Carlos, el informe de arribo se hace por medio del "Aviso de arribo de embarcaciones menores de 10 toneladas de registro bruto". Este aviso debe realizarse tanto por permisionarios como por concesionarios después de la faena de pesca. El documento informa a la autoridad competente, muchas de las veces encarnada en la subdelegación u oficina de pesca del poblado, acerca de los volúmenes de captura obtenidos durante una jornada o viaje de pesca.

EAH, un líder pesquero de la región, nos hizo ver que muchas de las veces los informes de arribo son imprecisos en cuanto a la zona de pesca. Parte del problema recae en las omisiones que hace aquel que llena y da el visto bueno al Formato de Aviso de Arribo. Cuando los pescadores no tienen una escolaridad tal que les permita llenar el formato de forma rápida, es normalmente el representante de CONAPESCA quien llena los formatos. En el caso de la pesquería de escama, los pescadores de San Carlos viajan hasta los bajos en el GdU y al tiempo de llenar el formato, el funcionario asume que la captura se realizó en la BMA. Con ello, los pescadores que realizan sus actividades en bajos del GdU como el Tetis y bancos como el 38, o el 23, tienen un registro de captura, señalado, erróneamente, fuera del GdU y esto deja su actividad económica fuera de cualquier reconocimiento que les pueda hacer acreedores a una compensación económica derivada de la moratoria pesquera.

Otra de las razones por las cuales la información del Formato de Aviso de Arribo es errónea recae en el hartazgo que sufren los pesca- 
dores a consecuencia de lo largo y pesado de las faenas de pesca. A medida que los volúmenes y calidad de la pesca han sido mermados en BMA, los pescadores han tenido que ir extendiendo la distancia y duración de sus expediciones pesqueras, muchas de las veces al amparo de infraestructura inadecuada. EAH lo resume de la siguiente manera:

Las embarcaciones de nosotros pues estamos tiempo de atrasados pues no tenemos la embarcación adecuada como para el riesgo que sufre. Se pone la vida, se van hasta 70-80 millas adentro para ir a pescar 3-4 días que ahí tiene que dormir, y tiene que hacer alimentos y tiene que estar arriba de la embarcación de este tipo pues, [algunas], como ésta ya están de alguna manera modernizada, pero las otras embarcaciones que se tienen a veces no cuentan ni con [uso real] para andar en alta mar.

Cuando se está en un estado de tensión y cansancio general, es difícil tener el ánimo de llenar el Formato de Aviso de Arribo, por lo que se llena con información dada a la ligera y sin una conciencia verdadera de las repercusiones a largo plazo que puede acarrear el registro, como es el caso del no acceso a las compensaciones derivadas de la moratoria pesquera.

Hay quienes, como GA, con quien platicamos durante la cena en nuestro último día en San Carlos, opinan que el mal manejo de los registros de arribo es un tema tan delicado que no conviene indagarlo muy a fondo, pues, tanto pescadores como autoridades juegan con las cantidades de arribo y sitios de captura para blanquear el producto furtivo, dando entrada al mercado al producto de los huateros.

\section{Opciones sustentables en San Carlos}

Una de las actividades que fácilmente podría suplir a la pesca, tanto en términos de ocupación como en nivel de ingresos, es la acuicultura. ZG, uno de los primeros maricultores y un notable emprendedor de San Carlos nos habló del creciente éxito de la empresa de producción de ostiones de la que participa junto con sus familiares, diciendo: "Tenemos un comprador en [Ciudad] Constitución... 
Cinco mil, ocho mil y hasta diez mil [ostiones] por semana nos empezó a comprar. Ayer nos habló, que quiere 4,500-5,000 ostiones por semana. Ya nosotros vamos a empezar a cosechar para ellos".

Otros actores, como EAH, también ven la acuicultura como una opción viable para salir de la situación que vive la pesquería en San Carlos. Él comenta:

La acuacultura también es algo en que nosotros creemos puede ser algo muy importante que se desarrolle en estas agua de Bahía Magdalena porque su calidad, precisamente hablando de los manglares, de los esteros llenos de mangle, son zonas donde por naturaleza se dan muchas especies, que es el camarón, el caracol, la jaiba lo que busques, por decir así, en esta zona y pues creo que se puede aprovechar...

No obstante, ve en la implementación de la acuicultura un posible conflicto por el espacio, alegando que la pesca ya está bien establecida en muchas áreas, tanto de la Bahía como en la boca del estero.

Parte fundamental de los problemas potenciales que puede traer consigo la adopción de una acuicultura de gran escala radica en la contaminación que se genera a través de los excedentes de comida, la excreción de los organismos y la propia respiración ( $c f r$. Wu 1995). No obstante, las técnicas de cultivo integral, que desde hace más de 10 años han resultado en producciones sostenidas y negocios rentables, se presentan como una buena opción para reducir la contaminación acuícola (e.g., Neori, Shipgel y Ben-Ezra, 2000; Neori et al., 2004) que permitiría producir peces, abulón, algas y otros moluscos en un esquema que emula de mejor manera que las granjas sencillas al productivo ecosistema de BMA.

El turismo es otra de las actividades que, poco a poco, se ha ido incorporando a la vida de los habitantes de Puerto San Carlos. Uno de los autores (DRA) comparte su experiencia y comenta: "Si, tú llevas y al turista ya le estás cobrando por salir nada más. Y además es divertido; y he llevado a ver las ballenas a familiares míos que vienen, es una actividad divertida, con eso gano dinero y hasta me gusta verlas, [cosa] que no había pensado antes". 
El turismo activo también ha sido desarrollado, poco a poco, en San Carlos. ZU nos platicó acerca del buceo con tiburones y la realización del documental "México Pelágico", tareas que, además de ofrecer actividades económicas no extractivas, dotan de un orgullo particular a los pescadores. Él comenta:

Empezamos proyectos nuevos con gente de la asociación "Pelagic Life". Ellos quieren nadar con tiburones... En un principio ellos venían buscando la bolas de carnada, con marlín, atunes, dorado... Andando allá afuera nos encontramos a los tiburoneros... Les platiqué que eran tiburoneros, que estaban pescando tiburones y dijeron: A ver vamos; y ya vieron y se aventaron. Había un tiburón enganchado, muerto, y dijeron: ¿ Tienen más? - Sí, les digo. Pues vamos y había uno vivo, y dijeron: que va hacer. No pues me voy arrimar y le voy a pegar un batazo para subirlo a la lancha muerto, porque son peligrosos. - No, no, espérate, diles que si nos lo venden. ¿Qué cuánto cuesta vivo, cuánto cuesta en el mercado? Y le digo: ¿Oye, más o menos cuánto cuesta ese tiburón? Ya si le sacas la aleta, unos MEX $\$ 150 \ldots$ esta chiquito el tiburón. Te vamos a dar MEX\$300, pero déjanos liberarlo; - Pues sale dice, después de esta carnada nomas ahorita que lo suelten vamos a seguirlo. ¡Zaz! Y ya, se fueron y me quedé con una carnada... se aventaron y lo soltaron, fotos y ya lo soltaron... ¡No, felices los chamacos!

Otra alternativa, proponen los pescadores de escama, sería una reducción temporal del esfuerzo pesquero en la captura de camarón en el interior del complejo lagunas. Es decir, durante al menos una temporada, que va de septiembre hasta marzo, utilizar sólo 50 \% del número de embarcaciones en operación Lo anterior con la finalidad de promover la conservación del recurso y hacer de esta pesquería una actividad económicamente rentable. Casos similares dejan ver que los pescadores de camarón siempre se mostrarán en desacuerdo con las moratorias pesqueras por razones económicas, sociales y ambientales (Musiello-Fernandes, Antunes y Hostim-Silva 2017).

Por otra parte, existe actualmente un proceso tendiente a la modificación de la Norma Oficial Mexicana NOM-002-SAG/PESC 2013, que regula la pesquería de camarón. La modificación consiste 
en permitir el acceso a la pesca de este recurso a las embarcaciones menores en la zona litoral del Golfo de Ulloa, esto, con el fin de disminuir la presión por pesca en el interior de la BM. Otra alternativa consiste en promover nueva, más robusta y mejor articulada normatividad (NOMS o acuerdos vía DOF) para la protección de las especies de callo de hacha y almeja generosa. Primero, con la finalidad de recuperar las poblaciones naturales. Segundo, para evitar una eventual sobreexplotación del recurso.

Sumado al estado de indefensión de los pescadores de escama ante la no compensación, sean estos libres o cooperativistas, el bienestar social de Puerto San Carlos también se ve vulnerado por procesos de delincuencia y adicción. En otras poblaciones pesqueras del noroeste del país la disponibilidad de estupefacientes aumenta durante la temporada cuando la pesquería está cerrada (Rentería-Valencia 2007), situación también manifiesta en Puerto San Carlos. DRA, dado su carácter de autor residente, hace ver que la baja de ingresos a lo largo del cierre de la temporada es indicador de que las pangas, los motores y cualquier otro equipo mal asegurado será sujeto a robo. Esto evidencia una situación difícil en tanto a la disposición para invertir trabajo y dinero en empresas como la acuicultura, pues tanto equipo como mariscos pueden ser robados antes de que deriven en una ganancia.

\section{DisCUSIONES Y CONCLUSIÓN}

A lo largo de 200 años, la abundancia de recursos en Baja California Sur ha sido vista desde una lógica extractiva que invita al saqueo de los recursos naturales mediante el uso de la mejor tecnología disponible. Esta visión de un desarrollo a ultranza, donde el ser humano logra colonizar, conquistar e imponerse al medio, recuerda el orientalismo ambiental de Gísli Pálsson (1996), un epistema que define a la naturaleza como una tierra inhóspita que debe de ser doblegada, transformada y domesticada de tal manera que genere un paisaje fácilmente reconocible y explotable para quien viene de otro lado.

Esta lógica orientalista, que intenta llevar a la naturaleza de lo crudo a lo cocido (sensu Levi-Strauss 1971), termina por generar un 
desajuste negativo en la economía energética de los ecosistemas, desajuste que repercute en la esfera social, que causa violencia ambiental (sensu Narchi 2015).

El ocaso de la abundancia en los recursos pesqueros en BMA y su consecuencia indirecta, la moratoria pesquera en el GdU, son un ejemplo más de las consecuencias que deja la evolución del orientalismo ambiental. A partir de la transformación del Noroeste de México con la introducción del sistema misional, la introducción del trigo y el ganado ( $c f r$. Narchi et al., 2015) y la reducción de los grupos de cazadores-recolectores, el paisaje se resignifica. Así, el ambiente xérico es tratado como desierto, una suerte de malpaís yermo e inútil que debe de ser modificado para que se vuelva útil y productivo. Esta misma lógica se magnifica con la entrada a la modernidad y la introducción de nuevas tecnologías que mediante la perforación de pozos, cada vez más profunda, logra impactar el sistema de acuíferos de los valles agrícolas hasta un punto en que colapsa la agricultura. A partir de ese momento y dadas las limitadas opciones que el árido ambiente ofrece, los campesinos y jornaleros se vuelcan a la costa y encuentran en la pesca la única esperanza de bienestar económico, desempeñándose, la mayoría de éstos, como pescadores de pequeña escala.

Uno de los problemas más grandes que trae consigo la migración desde la agricultura hacia la pesca es la falta de conocimiento ecológico. Antes hemos señalado que uno de los problemas fundamentales en las pesquerías de BMA, y de la pesca ribereña en general, encuentra fundamento en el régimen de acceso libre, régimen que permite un desarrollo de "la tragedia de los comunes". Este régimen colapsa muchas de las pesquerías que históricamente han sido el sustento económico de la región. No obstante, el colapso de los recursos debe de entenderse desde la historia de vida de los pescadores, otrora agricultores, y no, como postula Hardin (1968), por que los comunes están destinados a su colapso desde el inicio.

Todo modo de subsistencia tiene un conocimiento ecológico asociado. Este conocimiento depende, por fuerza, de prácticas sociales y culturales que han sido moldeadas a partir de costumbres e instituciones desde las cuales se incorpora una cosmovisión (Berkes 
et al., 2000). Antes, se ha sugerido que este conocimiento no requiere, por fuerza, una acumulación multigeneracional de experiencia (Narchi et al., 2014). En otras palabras, los individuos pueden acumular conocimiento ecológico local durante el curso de su propia vida, mientras interactúan con un ambiente local y tal conocimiento empírico puede ser compartido dentro de la comunidad en una misma generación.

Durante nuestra estancia en San Carlos fuimos testigos del vasto conocimiento ecológico local del que son poseedores los pescadores, quienes conocen las corrientes y la batimetría, reconocen las temporadas de pesca y los hábitos de los animales marinos que moran en sus localidades, sean o no estos organismos de importancia comercial. No obstante, creemos que la abundancia de recursos que históricamente han poblado BMA y el GdU, combinada con la depresión económica y problemática social que trajo consigo el colapso agrícola, sumado a las ganancias extraordinarias que la pesca representó de súbito para los nuevos pescadores y a la muy incipiente noción de ciencia pesquera que en ese entonces se tenía en el país, ${ }^{6}$ formaron una combinación de factores que permitió la sobreexplotación por encima de la compartición de un conocimiento tradicional que permitiese una explotación sostenida. Ello ha mermado cada uno de los stocks pesqueros. NAT lo resume muy bien:

Ahorita ya tengo dos temporadas que no trabajo el camarón, pero más antes, me ponía a contar cuantos lenguados agarrábamos en cada arrastre. Hay uno que es chiquito y otro que crece y llegué a contar 50 lenguados por lance. Así los lenguaditos [señala unos $4 \mathrm{~cm}$ con sus dedos]. Ese lenguado si se deja que crezca y que se capture, digamos de 2 kilos... Son como 400 lances que hacen los cargadores. Están matando más de 200 kilos de lenguado, no hacemos un plan de trabajo bien hecho.

La percepción de los pescadores acerca del estado actual de las pesquerías y su aparente disposición a hacer una transición hacia

${ }^{6}$ La primer institución de educación nacional que se ofreciera a formar profesionistas del mar es fundada en 1960 (Narchi y Aranda 2009). 
otro tipo de pesca e inclusive hacia otras actividades económicas como el turismo y la acuicultura se han hecho patentes durante nuestra estancia en San Carlos. No obstante, la falta de datos sistemáticos y de personal que entienda la problemática local, que esté capacitado y dispuesto a dar un acompañamiento integral a esta transición, a la vez que se adopta una política pesquera actual y de vanguardia, capaz de integrar nuevos y más complejos paradigmas (véase Hughes et al., 2005) en donde se vinculen los complejos procesos biofísicos del stock pesquero, los anhelos, necesidades e impulsos socioeconómicos del sector y los sistemas de gobernanza y conocimiento local que determinan el uso y aprovechamiento de los recursos marinos, hace que la tarea sea imposible sin un interés genuino por parte de todos los actores, entre los que destacan los tomadores de decisiones.

La moratoria pesquera en el Golfo de Ulloa no es un remedio a los problemas del deterioro del stock pesquero ni una solución eficaz para detectar la causa de mortandad de la tortuga amarilla o en el mejor de los casos, para asegurar su protección. La moratoria, junto con la afectación social y económica que ha causado en todos los actores locales del sector no es más que un reflejo del retroceso e inminente desmantelamiento del sector, planeado desde la disolución de la Secretaría de Pesca en 1994 (Cifuentes y Cupúl 2002; Alcalá 2003; Valiente et al., 2016). Lamentablemente, no podemos más que parafrasear lo dicho por EAH, quien afirma que la situación no puede cambiar "porque un dentista es el representante del sector pesquero. Por eso, 'ta cabrón”.

\section{Agradecimientos}

Agradecemos a las familias de Puerto San Carlos, Baja California Sur por su interés y participación. En especial a Adolfo González, excelente anfitrión. Agradecemos la ayuda de la doctora Micheline Cariño Olvera, el respaldo del Colegio de Michoacán y el apoyo de Erika Aguirre, Marina Chávez y Jesús Medina, así como los ricos comentarios de nuestros revisores. 


\section{Descargo DE RESPONSABILIDAD}

Nemer E. Narchi es secretario de redacción de la revista Relaciones Estudios de Historia y Sociedad desde enero de 2018. La publicación del presente manuscrito en la revista no constituye ningún conflicto de interés dado que fue propuesto para publicación en noviembre de 2016 y evaluado por pares en 2017, previo a su nombramiento como secretario. En ningún momento el doctor Narchi tuvo ingerencia en el proceso de evaluación y aceptación de su trabajo.

\section{REFERENCIAS}

AlCalá, Graciela. 2003. Políticas pesqueras en México (1946-2000). Contradicciones y aciertos en la planificación de la pesca nacional. Zamora: El Colegio de Michoacán. El Colegio de México, Centro de Investigación Científica y de Educación Superior de Ensenada.

Bar-Matthews, M. y C.W. Marean et al., 2010. "A High Resolution and Continuous Isotopic Speleothem Record of Paleoclimate and Paleoenvironment from 90 to $53 \nmid$ Ka from Pinnacle Point on the South Coast of South Africa". Quaternary Science Reviews 29(17-18): 2131-2145.

Berkes, Fikret, Johan Colding y Carl FolKe. 2000. "Rediscovery of traditional ecological knowledge as adaptive management". Ecological applications 10(5): 1251-1262.

BizzARRo, Joseph J. 2008. "A review of the physical and biological characteristics of the Bahia Magdalena lagoon complex (Baja California Sur, Mexico)". Bulletin, Southern California Academy of Sciences 107(1): 1-24.

Carocci, Fabio, Gabriella Bianchi, Paul Eastwood y Geoff Meaden. 2009. "Geographic Information Systems to support the ecosystem approach to fisheries". Roma: FAO Fisheries and Aquaculture Technical Paper, núm. 532, 120 p.

Cariño Olvera, Martha Micheline. 2011. "La identidad oasiana". En Oasis: Agua, biodiversidad y patrimonio, ed. Antonio Ortega Santos y Ana Molina Aguado. Granada: Editorial Atrio. 
Chávez Ortiz, J. Trinidad. 2007. "Los vencedores del desierto: formación de una ideología regional dominante". Imaginales Revista de Investigación Social (5) (enero-junio): 101-114.

Cifuentes, Juan Luis y Fabio Germán Cupui-Magaña. 2002. “Un vistazo o lo historía de la pesca en México: administración, legislación y esfuerzos para su investigación”. CIENCIA ergo sum 9(1):112-118.

Cota-Nieto, J. José, Victoria JimÉnez-Esquivel e Ismael MascaREÑAs-Osorio. 2016. "Fishing in Bahia Magdalena-Almejas: Economic Engine for Baja California Sur". DataMares. InteractiveResource, doi:10.13022/M39599 (Fecha de consulta: agosto de 2017).

Cruz Chávez, Gustavo Rodolfo y Osvaldo Ramírez Gómez. 2003. "Panorama agropecuario en Baja California Sur". En Diagnóstico estratégico de Baja California Sur. México. ed. Antonina Ivanova y Manuel Ángeles, 183-200. La Paz: Universidad Autónoma de Baja California Sur, Secretaría de Educación Pública, 183-200.

DOF (Diario Oficial de la Federación). 2015. "Acuerdo por el que se establece una zona de refugio pesquero y medidas para reducir la posible interacción de la pesca con tortugas marinas en la Costa Occidental de Baja California Sur”, México: Secretaría de Gobernación, 1-10.

2016. "Acuerdo por el que establece la zona de refugio pesquero y nuevas medidas para reducir la posible interacción de la pesca con tortugas marinas en la Costa Occidental de Baja California Sur", México: Secretaría de Gobernación, 1-14.

Funes Rodríguez, René, Jaime Gómez Gutiérrez y Ricardo PAlomares García. 2007. "Estudios Ecológicos en Bahía Magdalena”. La Paz: Centro de Investigaciones Biológicas del Noroeste S.C., Centro Interdisciplinario de Ciencias Marinas, Instituto Politécnico Nacional.

GÁmez, Alba E., Tamar Diana Wilson y Antonina Ivanova BonCHEVA. 2010. "Las mujeres en la migración interna y el empleo informal en Baja California Sur, México". La ventana. Revista de Estudios de Género 4(32): 214-243. 
García-Martínez, Salvador. 2005. "Análisis de estrategias para el manejo sostenible de la pesquería de camarón en Bahía Magdalena, Baja California Sur". Tesis de Doctorado, Universidad Autónoma de Baja California Sur, La Paz.

GArdner, Susan C. y Wallace J. Nichols. 2001. "Assessment of sea turtle mortality rates in the Bahía Magdalena region, Baja California Sur, México". Chelonian Conservation and Biology 4(1): 197-199.

GatTi, Luis María. 1986. Los pescadores de México: la vida en un lance. Serie: Los pescadores de México, vol. 110. México: Ciesas. Gobierno del Estado de Baja California Sur. 2008. Estadisticas municipales microrregiones, Secretaría de Promoción y Desarrollo Económico, La Paz: Centro Estatal de Información.

Godoy, Emilio. 2015. "México en riesgo de un embargo pesquero por tortugas caguama”. Proceso (14 de agosto). http://www.proceso.com.mx/412981/mexico-en-riesgo-de-un-embargo-pesquero-por-tortugas-caguama (Fecha de consulta: 12 de octubre de 2016).

Gómez Gallardo, Enrique, Salvador Lluch Cota, Felipe Melo Barrera y Alejandro Gómez Gallardo. 2014. Informe final de investigación: Estudio sobre las causas de muerte de la tortuga amarilla (Caretta Caretta) en la costa occidental de Baja California Sur, Golfo de Ulloa, La Paz: UABCS, CICIMAR-IPN, CIBNOR.

Handwerker, Penn W. 2001. Quick Ethnography: A Guide to Rapid Multi-Method Research. Plymouth: Altamira Press.

Hardin, Garrett. 1968. "The Tragedy of the Commons". Science 162(3859): 1243-1248.

Hewitt de Alcántara, Cynthia. 1976. Modernizing Mexican Agriculture: Socioeconomic Implications of Technological Change, 1940-1970. Genova: United Nations Research Institute for Social Development.

Hughes, Terence P., David R. Bellwood, Carl Folke, Robert S. Steneck y James Wilson. 2005. "New Paradigms for Supporting the Resilience of Marine Ecosystems". Trends in Ecology \& Evolution 20(7): 380-386.

Instituto Nacional de Estadística y Geografía. 2013. Censo 
de Población y Vivienda 2010, México: Instituto Nacional de Estadística Geografía.

- Sintesis Geográfica del estado de Baja California Sur. 1995. La Paz: Instituto Nacional de Estadística y Geografía, Gobierno del Estado de Baja California Sur.

Koch, Volker, Wallace Nichols, Hoyt Peckham y Victor De LA Това. 2006. "Estimates of Sea Turtle Mortality from Poaching and Bycatch in Bahia Magdalena, Baja California Sur, Mexico". Biological Conservation 128(3): 327-334.

LeVi-Strauss, Claude. 1971. "Lo crudo y lo cocido". Revista de la Universidad Nacional (1944-1992) (9): 119-157.

Maldonado, David, Aarón Esliman, Juan Rodríguez y Hoyt Peckham. 2012. Frecuencia extraordinaria de varamientos de la tortuga amarilla (Caretta Caretta) en playa San Lázaro BCS durante el periodo 1 de enero-22 de mayo de 2012, Actualización del reporte presentado en enero de 2012 por el Grupo Tortuguero de las Californias AC.

Marean, C.W., M. Bar-Matthews, J. Bernatchez, E. Fisher, P. Goldberg, A.I.R. Herries, Z. Jacobs, A. Jerardino, P. Karkanas, T. Minichillo, P.J. Nilssen, E. Thompson, I. Watts y H.M. Williams. 2007. "Early Human Use of Marine Resources and Pigment in South Africa during the Middle Pleistocene". $\mathrm{Na}$ ture 449(7164): 905-908.

MÉndez García, Ulises, 2005. "Bases para el manejo sustentable: evaluación de la pesca artesanal en el complejo lagunar Santo Domingo-Magdalena-Almejas, B.C.S., México”. Tesis de Maestría, Centro de Investigaciones Biológicas del Noroeste, S.C.

Musiello-Fernandes, Joelson, Camila Antunes Zappes y Mauricio Hostim-Sinva. 2017. "Small-Scale Shrimp Fisheries on the Brazilian Coast: Stakeholders Perceptions of the Closed Season and Integrated Management". Ocean \& Coastal Management, vol. 148 (noviembre): 89-96.

NarCHI, Nemer E. 2015. "Environmental Violence in Mexico a Conceptual Introduction". Latin American Perspectives 42(5): 5-18.

Narchi, Nemer E. y Francisco Javier Aranda-Manteca. 2009. "Arenas del Tiempo: El ritual como mecanismo de conforma- 
ción de la identidad profesional entre los estudiantes de oceanología de la Universidad Autónoma de Baja California, México”. Ecological and Environmental Anthropology 5(1): 39-66.

Narchi, Nemer E., Alberto Búrquez, Sarah Trainer y Rodrigo F. Rentería-Valencia. 2015. "Social Constructs, Identity, and the Ecological Consequences of Carne Asada". Journal of the Southwest 57(2): 305-336.

Narchi, Nemer E., Samuel Cornier, Donata Melaku Canu, Luis E. Aguilar-Rosas, Mariana G. Bender, Christian Jacquelin, Marion Thiba, Gustavo GM Moura y Rutger De Wit. 2014. "Marine Ethnobiology a Rather Neglected Area, Which Can Provide an Important Contribution to Ocean and Coastal Management". Ocean \& Coastal Management, vol. 89 (marzo): 117-126. Narchi, Nemer E., Alberto Búrquez, Sarah Trainer y Rodrigo F. Rentería-Valencia. 2015. "Social Constructs, Identity, and the Ecological Consequences of Carne Asada". Journal of the Southwest 57(2): 305-336.

Neori, Amir, Muki ShPigel y David Ben-Ezra. 2000. "A Sustainable Integrated System for Culture of Fish, Seaweed and Abalone". Aquaculture 186(3): 279-291.

Neori, Amir, Thierry Chopin, Max Troell, Alejandro H. Buschmann, George P. Kraemer, Christina Halling, Muki Shpigel y Charles YARISH. 2004. "Integrated Aquaculture: Rationale, Evolution and State of the Art Emphasizing Seaweed Biofiltration in Modern Mariculture". Aquaculture 231(1): 361-391.

Nichols, Wallace J., Antonio Resendiz, Jeffrey A. Seminoff y Beatrice Resendiz. 2000. "Transpacific Migration of a Loggerhead Turtle Monitored by Satellite Telemetry”. Bulletin of Marine Science 67(3): 937-947.

PÁlsson, Gísli. 1996. "Human-environmental Relations”. En $\mathrm{Na}$ ture and Society: Anthropological Perspectives, ed. Philippe Descola y Gísli Pálsson, 63-81. Londres: Taylor \& Francis.

Peckham S. Hoyt, David Maldonado Díaz, Andreas Walli, Georgita Ruiz, Larry Crowder y Wallace Nichols. 2007. "Small-scale fisheries bycatch jeopardizes endangered Pacific loggerhead turtles". PLOS ONE 2(10): e1041. 
Peckham, H. S., David Maldonado Díaz, V. Koch, A. Mancini, A. Gaos, M. Tim Tinker y W. J. Nichols. 2008. "High Mortality of Loggerhead Turtles Due to Bycatch, Human Consumption and Strandings Baja California Sur, Mexico, 2003 to 2007”. Endangered Species Research 5(2-3): 171-183.

Ramírez Rodríguez, Mauricio, Gustavo de la CRUz, Elvia MARÍN, Miguel Ángel Ojeda y German Ponce. 2010. Estudio sobre la caracterización socioeconómica y pesquera del área del Golfo de Ulloa, Baja California Sur, Informe Técnico Final del Proyecto. La Paz: Centro Interdisciplinario de Ciencias Marinas del Instituto Politécnico Nacional.

Rentería Valencia, R. Seris. 2007. México: Comisión Nacional para el Desarrollo de los Pueblos Indígenas.

Resendiz, Antonio, Beatris Resendiz, Wallace J. Nichols, Jeffrey A. Seminoff y Naoki Kamezaki. 1998. "First Confirmed EastWest Transpacific Movement of a Loggerhead Sea Turtle, Caretta caretta, Released in Baja California, Mexico". Pacific Science 52(2): 15-153.

SAgarpa (Secretaría de Agricultura, Ganadería, Desarrollo Rural, Pesca y Alimentación). 2014. Programa Integral de Ordenamiento pesquero en el Golfo de Ulloa, Baja California Sur, Secretaría de Agricultura, Ganadería, Desarrollo Rural, Pesca y Alimentación. México: Comisión Nacional de Acuacultura y Pesca.

2016a. Programa de Fomento a la Productividad Pesquera y Acuicola. Convocatoria para dar a conocer las bases para acceder a los incentivos del "Proyecto estratégico para implementar acciones que contribuyan a mitigar el efecto de la restricción normativa en la actividad pesquera en la región del Golfo de Ulloa, Baja California Sur". México: SAGarpa.

2016b. Hoy inicia veda en Golfo de Ulloa, Comunicado de Prensa. México: Secretaría de Agricultura, Ganadería, Desarrollo Rural, Pesca y Alimentación, BCS/275/2016.

Stiner, M. 1994. Honor among Thieves: A Zooarchaeological Study of Neandertal Ecology. Princeton: University Press Princeton.

Valiente, Carmina, Micheline Cariño Olvera, Néstor Corona Morales y Nemer E. NARCHI. 2016. "Despojo, riesgo y vulne- 
rabilidad: Consecuencias (no) deseadas del desarrollo inmobiliario en Baja California Sur, México". Ecología política (51):79-83. Wolcotт, Harry F. 1999. Ethnography: A way of seeing. Lanham: Altamira Press.

Wu, R. S. S. 1995. "The Environmental Impact of Marine Fish Culture: Towards a Sustainable Future". Marine Pollution Bulletin 31(4): 159-166.

Young, Emily H. 1999. "Balancing Conservation with Development in Small-Scale Fisheries: Is Ecotourism an Empty Promise?" Human Ecology 27(4): 581-620.

. 2001. "State Intervention and Abuse of the Commons: Fisheries Development in Baja California Sur". Annals of the Association of American Geographers 91(2): 283-306. 